\title{
Vapor transport within the thermal diffusion cloud chamber
}

\author{
Frank T. Ferguson \\ Richard H. Heist \\ Fairfield University, rheist@fairfield.edu \\ Joseph A. Nuth III
}

Follow this and additional works at: https://digitalcommons.fairfield.edu/engineering-facultypubs Copyright 2000 American Institute of Physics

The final publisher PDF has been archived here with permission from the copyright holder.

\section{Peer Reviewed}

\section{Repository Citation}

Ferguson, Frank T.; Heist, Richard H.; and Nuth, Joseph A. III, "Vapor transport within the thermal diffusion cloud chamber" (2000). Engineering Faculty Publications. 140.

https://digitalcommons.fairfield.edu/engineering-facultypubs/140

\section{Published Citation}

Ferguson, F. T., Heist, R. H., \& Nuth III, J. A. (2000). Vapor transport within the thermal diffusion cloud chamber. The Journal of Chemical Physics, 113(17), 7398-7405. doi:10.1063/1.1312381.

This item has been accepted for inclusion in DigitalCommons@Fairfield by an authorized administrator of DigitalCommons@Fairfield. It is brought to you by DigitalCommons@Fairfield with permission from the rightsholder(s) and is protected by copyright and/or related rights. You are free to use this item in any way that is permitted by the copyright and related rights legislation that applies to your use. For other uses, you need to obtain permission from the rights-holder(s) directly, unless additional rights are indicated by a Creative Commons license in the record and/or on the work itself. For more information, please contact digitalcommons@fairfield.edu. 


\title{
Vapor transport within the thermal diffusion cloud chamber
}

\author{
Frank T. Ferguson \\ Department of Chemistry, Catholic University of America, Washington, DC 20064 \\ Richard H. Heist \\ Department of Chemical Engineering, Manhattan College, Riverdale, New York 10471-4098 \\ Joseph A. Nuth III \\ Code 691, NASA-Goddard Space Flight Center, Greenbelt, Maryland 20771
}

(Received 23 March 2000; accepted 2 August 2000)

\begin{abstract}
A review of two different, one-dimensional models of the vapor transport within the thermal diffusion cloud chamber (TDCC) is presented. In one case the assumption is made that there are no convective fluxes within the chamber and that heat and mass transport occur by diffusion only. Although in this model there are no restrictions on the transport of the two components within the chamber, the assumption of no velocities within the chamber results in an incorrect flux boundary condition for the background, carrier gas. The second model is based on the typical, stagnant background gas assumption and the equations of this model closely follow those of the classical Stefan tube problem in which there is transport of a volatile species through a noncondensible, carrier gas. Unfortunately, this model of the TDCC also suffers from the same inconsistencies as noted by several researchers for the Stefan tube. When the convective contributions to the flux are low in the stagnant background gas model, the two models give reasonably close results. For more convective situations, the supersaturation results can differ by more than $50 \%$. One interesting feature of the zero velocity model is that it predicts a change in the supersaturation profile with pressure, whereas no pressure dependence is predicted with the stagnant background gas model. Unfortunately, the direction of this pressure change is opposite to that seen in experimental observations. (C) 2000 American Institute of Physics. [S0021-9606(00)50741-7]
\end{abstract}

\section{INTRODUCTION}

The thermal diffusion cloud chamber (TDCC) has been used to study the nucleation of a variety of materials since its introduction to the nucleation community by Katz and Ostermeir over 30 years ago. ${ }^{1}$ In the early years, it was used to measure the critical supersaturation, $S_{\mathrm{cr}}$, the supersaturation at which the flux of droplets was approximately $1 \mathrm{~cm}^{-3} \mathrm{~s}^{-1}$. In the late 1980s as researchers began measuring both the flux as well as the supersaturation, they noticed a dependence of the flux on the background gas that was not seen in typical expansion studies. ${ }^{2}$ Since a difference of a few percent in the supersaturation can cause an order of magnitude or more difference in the flux, these flux measurements were extremely sensitive indicators of differences between the two experimental systems.

To examine the role of the background gas on the nucleation behavior, Heist et al. developed a high pressure diffusion cloud chamber (HPCC) capable of studying the nucleation of materials at pressures as high as 40 bar. $^{3-5}$ The results of this work seem to suggest that there is a significant pressure dependence of the critical supersaturation, increasing with increasing pressure.

Today there is increasing concern in determining the stable range of operation of the TDCC/HPCC. For example, at sufficiently high pressures, there can be an inversion in the density profile. Such an inversion results in strong convective currents within the chamber, thereby rendering the 1D model of the TDCC system invalid. A more insidious prob- lem can be caused by convective flows generated by sidewall buoyancy effects. ${ }^{6}$ In this case, convective flows can cause a slight, yet systematic change in the temperature and mole fraction profiles within the chamber. Because of their small magnitude, it may be difficult to detect such flows experimentally.

To examine the possible magnitudes of these flows, Ferguson and Nuth developed a two-dimensional model of a typical diffusion cloud chamber which includes the appropriate buoyancy effects. ${ }^{7}$ Ferguson and Nuth found that small flows can exist and, for the cases they investigated, these flows were able to reduce the maximum supersaturation along the center line by roughly $2-8 \%$.

The equations used to solve for the temperature and concentration fields are coupled and depend upon the expressions used for the physical properties of the constituent species. In order to clearly delineate the effects due to buoyancy alone, the authors calculated the maximum supersaturation within the chamber with the model at a gravitational level of 1 and 0 . With $g=0$, buoyancy effects are eliminated and the results should be identical to the typical one-dimensional (1D) modeling [provided the diameter to height ratio $(D / H)$ is sufficiently high and that wall effects do not extend to the center line].

Although very close, the $0 \mathrm{~g}$ solution and that of the typical 1D modeling did not match identically, even using identical physical properties. The following discussion outlines the differences between these two approaches and tries 


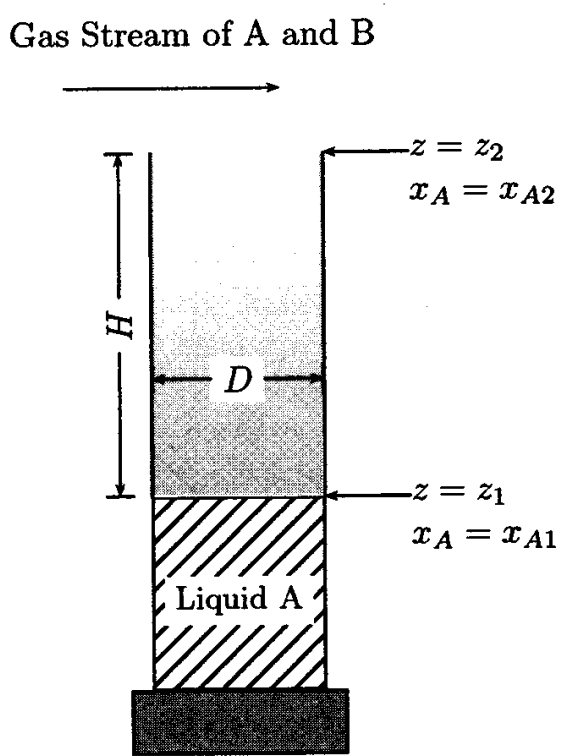

FIG. 1. Cross-sectional diagram and mass transfer boundary conditions for the classical Stefan tube problem.

to emphasize the importance of the momentum boundary condition in the modeling of the TDCC.

\section{MODELING OF THE TDCC}

The development of the equations for the TDCC follow very closely those for the classical Stefan tube problem as outlined by Bird, Stewart, and Lightfoot. ${ }^{8}$ Such an apparatus can be used to measure binary diffusion coefficients and a diagram of a typical Stefan tube apparatus is shown in Fig. 1. The tube is filled with a liquid $A$ evaporating into a background gas $B$ and it is assumed that the mole fraction at the liquid surface is given by the ratio of the equilibrium vapor pressure at the temperature of the liquid to the total pressure. At the top of the tube, the mole fraction of $A$ is also specified.

Fick's law for the transport of $A$ is

$$
\mathbf{N}_{\mathbf{A}}-x_{A}\left(\mathbf{N}_{\mathbf{A}}+\mathbf{N}_{\mathbf{B}}\right)=-c D_{A B} \nabla x_{A},
$$

where $\mathbf{N}_{\mathbf{i}}$ is the molar flux of species $i$ with respect to a fixed coordinate system, $c$ the molar concentration of the mixture, $D_{A B}$ the binary diffusion coefficient, and $x_{i}$ the mole fraction of species $i$. If we assume the flux is one-dimensional and that the background gas is essentially stagnant (i.e., $N_{B z}=0$ ) we get

$$
N_{A z}=-\frac{c D_{A B}}{1-x_{A}} \frac{d x_{A}}{d z} .
$$

If it is assumed that the tube is at steady state and isothermal and that the gases behave ideally, a shell balance for the flux of $A$ can be used to develop analytical expressions for the mole fraction profile and the rate of evaporation. ${ }^{8}$

A diagram of a typical diffusion cloud chamber and the boundary conditions for the typical 1D modeling are shown in Fig. 2. The TDCC differs from the Stefan tube in that the top boundary is closed and the bottom and top surfaces are held at different temperatures. During operation, vapor diffuses from the hotter, lower plate towards the cooler, upper

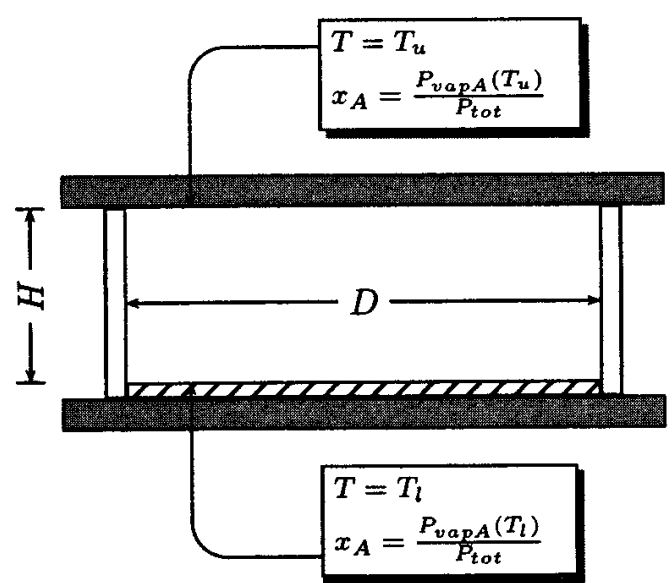

FIG. 2. Typical thermal diffusion cloud chamber and transport boundary conditions.

plate. At sufficiently high supersaturations the vapor condenses and forms droplets which fall back to the lower plate. In order to model the system in one-dimension only, the $D / H$ ratio of such chambers are typically large, approximately 5 or greater. In contrast, Stefan tubes typically have very low $D / H$ ratios to minimize end effects (e.g., error in fluid meniscus level, circulating flows at the top of the tube).

Katz originally developed the equations describing transport in the TDCC. ${ }^{9}$ Because the system is not isothermal, an additional equation is needed for the temperature profile. The mass flux in the $1 \mathrm{D}$ system is given by

$$
x_{B} N_{A z}-x_{A} N_{B z}=-c D_{A B}\left[\frac{d x_{A}}{d z}+k_{T} \frac{d}{d z} \ln T\right],
$$

where $k_{T}$ is the thermal diffusion coefficient and $T$ is the temperature. The second term in the brackets accounts for the influence of the temperature field on the mass flux, i.e., the Soret effect. Again the assumption is made that the flux of $B$ is zero; hence the equation can be written as

$$
x_{B} N_{A z}=-c D_{A B}\left[\frac{d x_{A}}{d z}+k_{T} \frac{d}{d z} \ln T\right] .
$$

It is more convenient to define the thermal diffusion ratio, $\alpha$, as

$$
\alpha=\frac{k_{T}}{x_{A} x_{B}}
$$

Also, the following substitution can be made for the mole fraction, $x_{A}$ :

$$
x_{A}=\frac{P}{P_{t}},
$$

where $P$ is the partial pressure of $A$ and $P_{t}$ is the total pressure within the chamber. The binary diffusion coefficient, $D_{A B}$, can be expressed as

$$
D_{A B}=\frac{D_{A B}^{0} T^{s}}{c}=\frac{D_{A B}^{0} R T^{s+1}}{P_{t}}
$$


where $D_{A B}^{0}$ is a constant and $s$ is a factor ranging from 0.5 to 1.0. Substituting these expressions into Eq. (4) yields a differential equation for the partial pressure profile of $A$ :

$$
\frac{d P}{d z}=\frac{\alpha P\left(P-P_{t}\right)}{T P_{t}} \frac{d T}{d z}+\frac{\left(P-P_{t}\right) L}{T^{s} D_{A B}^{0}} .
$$

The heat flux, $Q$, in the TDCC consists of three terms: transport by conduction, transport due to convective flux, and the Dufour effect. This flux is given by

$$
Q=-k \frac{d T}{d Z}+N_{A z} H+\frac{R T k_{T} N_{A z}}{x_{A}},
$$

where $k$ and $H$ are the thermal conductivity and enthalpy of the mixture, respectively. Using the same substitutions as used for the molar flux equation gives

$$
\frac{d T}{d Z}=\frac{1}{k}\left[-Q+N_{A}\left(H+\frac{\alpha R T\left(P_{t}-P\right)}{P_{t}}\right)\right] .
$$

The coupled differential equations (8) and (10), can be solved numerically for the temperature and partial pressure profile to determine the supersaturation profile.

\section{DIFFERENCES IN THE MODELING}

In contrast to the typical assumptions used to compute the concentration and temperature field, Ferguson and Nuth assumed that all velocity components at the chamber boundaries were zero. with the exception of the symmetry condition at the chamber center line. Under this assumption and in the absence of any pressure gradients and body forces, there is no generation of momentum within the chamber so that

$$
\mathbf{v}=0
$$

at every point within the chamber and the transport of mass and energy within the chamber is purely diffusional. No assumption was made on the transport of either the volatile species, $A$, or the background gas, $B$, within the chamber. In other words, the background gas, $B$, was not forced to be stagnant. Yet, the consequence of specifying a zero velocity at the boundaries is a nonzero flux of the insoluble, background gas at the solution boundaries. In general, for the 1D case the mass-average velocity within the chamber at any point is given by

$$
v_{z}=\frac{1}{\rho}\left[N_{A z} M_{A}+N_{B z} M_{B}\right]
$$

For a nonzero flux of $A, N_{A z}$, at the top and bottom boundaries, the assumption of $v_{z}=0$ implies that there is also a compensating flux of the background gas into the liquid at these boundaries. Therefore, there is an unrealistic boundary condition in the solution of such a model.

The equations typically used to calculate the concentration and temperature profiles in the TDCC have inconsistencies as well. If the assumption is made that the background gas is insoluble in the volatile liquid, i.e., $N_{B z}=0$ at the boundaries, then

$$
v_{z}=\frac{1}{\rho}\left[N_{A z} M_{A}\right]
$$

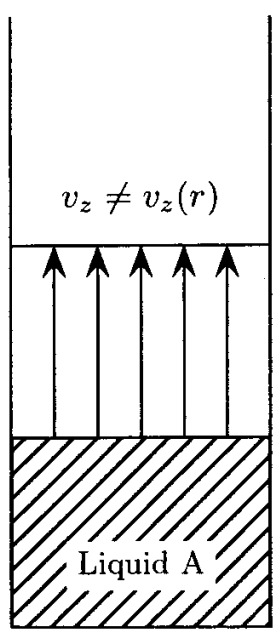

(a)

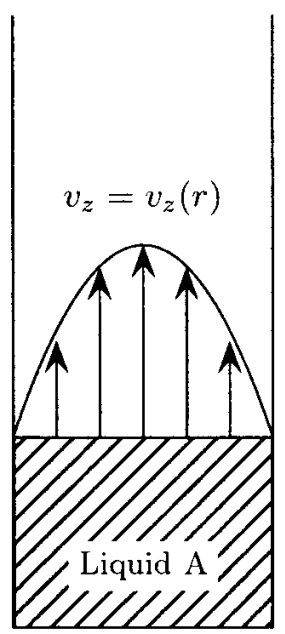

(b)
FIG. 3. Two different concepts of the velocity profile within the stefan tube and diffusion cloud chamber.

In a $1 \mathrm{D}$ model, if $N_{B z}=0$ at the boundaries, then by continuity the flux of background gas must also be zero throughout the chamber and this is the origin of the stagnant background gas assumption. Therefore Eq. (13) is not only valid at the boundaries but it must hold throughout the chamber. Under steady operation, the flux of $A$ is constant and Eq. 13 indicates that the velocity within the chamber varies inversely with the overall density. Under normal chamber operation the density decreases with height in the chamber. Therefore, the highest velocity occurs at the top of the chamber where the density is the lowest.

As suggested by Whitaker, one problem with Eq. (13) is that it must break down at high concentrations of the volatile species. ${ }^{10}$ For example, the velocity in Eq. (13) depends on the flux of $A$, which is given by Eq. (2) (if we neglect the Soret effect in this particular example). This equation, and hence the mass-average velocity within the chamber, suffers from problems as $x_{A} \rightarrow 1.0$. For the TDCC, this is generally not a problem since the mole fraction of the volatile species is typically low.

Another limitation of Eq. (13) is that there is no radial dependence in this equation for the velocity in the chamber and this equation predicts a flat velocity profile as shown in Fig. 3(a); yet such a profile violates the no-slip boundary condition at the chamber side wall. For laminar flow, the no-slip boundary at the chamber sidewall should give a parabolic-type of velocity profile similar to that shown in Fig. 3(b).

It was recognized quite a while ago that the uniform velocity profile violated the no-slip boundary condition at the wall and this resulted in a radial variation in the concentration gradient. Heinzelmann et al. performed a detailed experimental and theoretical analysis of the Stefan tube assuming no-slip at the walls and concluded that the radial concentration was essentially uniform within their experimental error. ${ }^{11}$ Rao and Bennet performed another study of radial concentration effects in the Stefan tube and arrived at a similar conclusion. ${ }^{12}$

Later on, justifications for the apparent discrepancy be- 
tween the velocity profile of species $A$ and the no-slip boundary condition came including the presence of slip at the walls. Kramers and Kistemaker ${ }^{13}$ postulated the existence of a diffusive slip boundary condition in the presence of a concentration gradient and this was invoked by Whitaker as a possible explanation for the momentum boundary condition inconsistency. ${ }^{14}$

Rao and Bennet later argued that the no-slip boundary condition could be satisfied if there was recirculation of the background gas. ${ }^{15}$ This $2 \mathrm{D}$ phenomena would be in contrast to the typical stagnant background gas assumption made in 1D. In order to satisfy the no-slip boundary condition at the walls (i.e., $v_{z}=0$ ), of the Stefan tube they envisioned a downward flux of the background gas in accordance with Eq. (12). This downward flux at the walls would have to be balanced by an upward flux of $B$ in the interior of the tube.

This postulation was first confirmed in a numerical simulation by Meyer and Kostin for a constant density situation, (i.e., components $A$ and $B$ of equal molecular weight). ${ }^{16}$ These authors showed that there was considerable recirculation of the background gas in contrast to the typical, "stagnant" approximation. Later Markham and Rosenberger made a much more general and refined numerical study of the Stefan tube problem. ${ }^{17}$ They considered unequal molecular weight species and examined the resulting concentration profiles as well as the velocity distribution within the tube. Like Meyer and Kostin, they also found considerable recirculation of the background gas. In addition, they also noted that the radial density profile depended upon the ratio of the molecular weights and that the total mass flux through the Stefan tube differs very little from the 1D solution for typical tube conditions where $H / D \sim 10$. They did warn that with a decrease in this ratio, buoyancy-driven contributions can develop that can cause larger errors (several 10\%) in the typical 1D solution. ${ }^{17}$

In summary we have presented two different models of the TDCC and each represents a limiting case of the 1D modeling. Each one of these models also suffers from some problems or inconsistencies. In the zero velocity model, no assumption is made about the transport of $A$ or $B$ within the chamber, but specification of a zero velocity at the boundary results in an incorrect flux boundary condition for the background gas. In the case of the stagnant background gas model, the no flux boundary condition of the background gas at the boundaries requires that $N_{B z}=0$ at all points within the chamber. The work of Meyer and Kostin and Markham and Rosenberger has shown that in fact there is considerable recirculation of this background gas. These inconsistencies in both models can be resolved by resorting to a $2 \mathrm{D}$ model of the chamber which contains elements of each of these 1D, limiting cases. Such a model is unfortunately much more complex and a 1D model is obviously preferred in the calculation of chamber supersaturation data.

In this paper we make a comparison between these two ID models and show the characteristics and predictions of each. To make such a comparison, we neglect the crosscoupling terms (Soret/Dufour effects) in the transport equations. The emphasis of this analysis is to highlight the differences between the terms describing ordinary, concentration- induced diffusion; the cross-coupling terms would unnecessarily complicate the analysis and may be included in a full analysis with little effort.

A mass balance on species $A$, at steady state and without generation terms through chemical reactions gives

$$
\boldsymbol{\nabla} \cdot\left(\rho \omega_{A} \mathbf{v}\right)=\left(\boldsymbol{\nabla} \cdot \boldsymbol{\rho} D_{A B} \boldsymbol{\nabla} \omega_{A}\right),
$$

where $\omega_{A}$ is the mass fraction of species, $A$. Since the velocities in the momentum and continuity equations are mass average velocities, there is an advantage to switching to mass fractions for concentrations rather than using mole fractions. In Eq. (14) for 1D, assuming $\mathbf{v}=0$ everywhere, the inertial term drops out and we are left with

$$
\frac{d}{d z}\left[\rho D_{A B} \frac{d \omega_{A}}{d z}\right]=0
$$

Using $\rho=c M$ where $M$ is the mean molecular weight of the mixture and

$$
\omega_{A}=\left[\frac{x_{A} M_{A}}{M}\right]
$$

in Eq. (15) gives

$$
\frac{d}{d z}\left[\frac{c D_{A B} M_{A} M_{B}}{M} \frac{d x_{A}}{d z}\right]=0 .
$$

Since $M_{A}$ and $M_{B}$ are constants we get

$$
\frac{d}{d z}\left[\frac{c D_{A B}}{M_{B}+\left(M_{A}-M_{B}\right) x_{A}} \frac{d x_{A}}{d z}\right]=0 .
$$

This equation should be compared with the equation under the stagnant gas approximation

$$
\frac{d}{d z}\left[\frac{c D_{A B}}{\left(1-x_{A}\right)} \frac{d x_{A}}{d z}\right]=0
$$

obtained from Eq. (2) by assuming the flux is constant throughout the chamber. As $x_{A} \rightarrow 0$, the denominator of Eq. (18) $\rightarrow M_{B}$. Since the denominator of Eq. (18) is just the mean molecular weight of the binary mixture, it is always greater than zero.

\section{COMPARISON BETWEEN EXPRESSIONS}

What is the difference in the mole fraction profiles between Eqs. (18) and (19)? To examine this effect we assume that the $c D_{A B}$ product is a constant (which is reasonable for an ideal gas mixture); then the equations reduce to a comparison of

$$
\frac{d}{d z}\left[\frac{1}{\left(1-x_{A}\right)} \frac{d x_{A}}{d z}\right]=0
$$

and

$$
\frac{d}{d z}\left[\frac{1}{M_{B}+\left(M_{A}-M_{B}\right) x_{A}} \frac{d x_{A}}{d z}\right]=0 .
$$

For the second equation we need molecular weights so we choose $M_{A}=60.096$ and $M_{B}=4.0026$ corresponding to 1-propanol and helium, respectively, as a test case.

Figure 4 is a comparison between these two equations for three different cases with a constant difference in the 


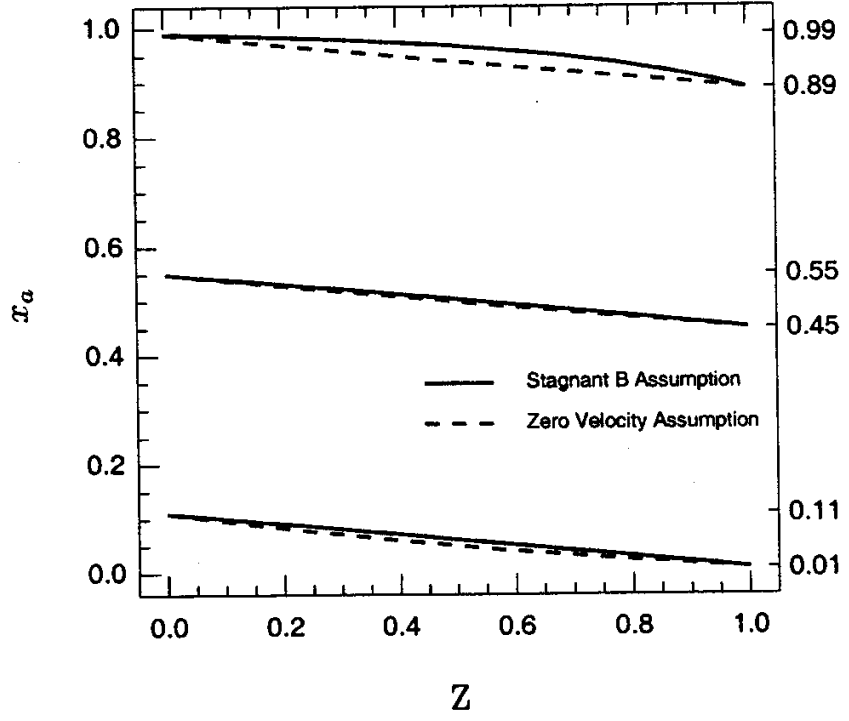

FIG. 4. A comparison between the predicted mole fraction profiles based on the stagnant gas assumption and the zero velocity assumption. Curves are shown for different concentrations with a total $\Delta x_{a}$ of 0.1 .

mole fraction at the boundaries, $\Delta x_{A}$, of 0.1 . The resulting profiles will depend upon the value of $x_{A}$ so three extreme cases are shown in the graph; $x_{A} \rightarrow 0, x_{A} \sim 0.5$, and $x_{A}$ $\rightarrow 1.0$. In each case, the actual mole fraction boundary conditions used in the computation are shown on the right-hand side of the figure. The dark curve denotes the typical, stagnant background gas solution while the dashed curve is the profile calculated using the zero velocity assumption.

In all three cases, Eq. (21) predicts a smaller value for the mole fraction than Eq. (20). For values of $x_{A} \sim 0.5$, there is virtually no difference between the two solutions. As $x_{A}$ $\rightarrow 0$ and $x_{A} \rightarrow 1.0$, the differences between the two profiles are larger and the effect is more pronounced for the $x_{A}$ $\rightarrow 1.0$ case.

Figure 5 is a similar plot for the mole fraction profile, but with a larger concentration difference between the

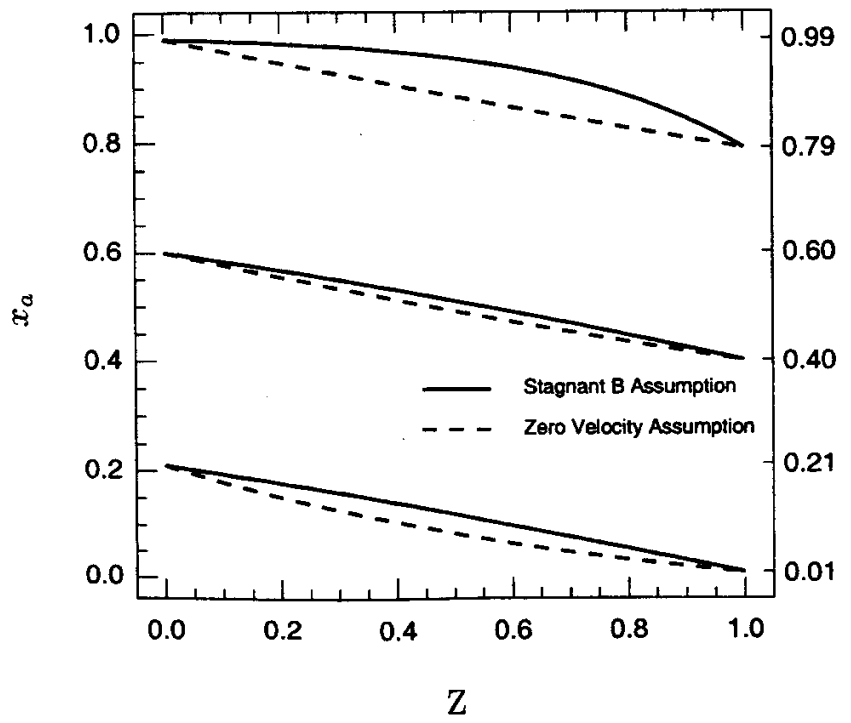

FIG. 5. A comparison between the predicted mole fraction profiles based on the stagnant gas assumption and the zero velocity assumption. Curves are shown for different concentrations with a total $\Delta x_{a}$ of 0.2 . boundaries. In this case, the concentration difference between the two boundary points is 0.2 . Again, in all cases the stagnant background gas approximation [Eq. (20)] predicts a higher value for the mole fraction of $A$ at any point over the results from Eq. (21).

The differences between the two profiles are again smallest for the $x_{A} \sim 0.5$ solution, but the differences between the solutions are more dramatic for the $\Delta x_{A}=0.2$ case than for the $\Delta x_{A}=0.1$ case.

Because of the coupling between the equations for the concentration and temperature profiles, it can be hazardous to draw general conclusions as to the results on the supersaturation profile. The results from Figs. 4 and 5 seem to suggest that the supersaturation calculated via Eq. (21) will always be lower than that calculated using Eq. (20) and that these differences will be more pronounced as the concentration of the vapor becomes very small or very large.

\section{CALCULATION OF SUPERSATURATION DATA}

To examine the effect of the stagnant background gas and zero velocity assumption on the actual supersaturations calculated, we will examine two test cases. The first of these will be the condensation of 1-propanol in helium at 1.18 bar with lower and upper plate temperatures of 302.9 and 256.5 $\mathrm{K}$, respectively. The maximum supersaturation between the two plates is calculated via the typical $1 \mathrm{D}$ equations derived by Katz and by the following two equations for the temperature and mass fraction profile, respectively:

$$
\begin{aligned}
& \frac{d}{d z}\left[k \frac{d T}{d z}\right]=0, \\
& \frac{d}{d z}\left[\rho D_{A B} \frac{d \omega_{A}}{d z}\right]=0 .
\end{aligned}
$$

These equations are based upon the zero velocity assumption in which there is purely diffusional transport of energy and mass within the chamber. As with the traditional equations for the TDCC, the condensation flux is assumed to be sufficiently small that the effect of the condensing vapor does not significantly influence the temperature or concentration profile as calculated by these equations. Physical properties for both sets of equations were identical and were taken from the tabulated data given by Heist. ${ }^{3}$ In both sets of equations the Soret and Dufour effects were neglected and the ideal gas equation of state was used.

The results were an $S_{\max }$ of 3.226 calculated via Eqs. (22) and (23) and an $S_{\max }$ of 3.66 as calculated via the typical, stagnant background gas solutions. The $S_{\max }=3.226$ is identical to the solution derived by the $2 \mathrm{D}$ model at $0 \mathrm{~g}$ by Ferguson and Nuth ${ }^{7}$ while the 3.66 value is close to the value estimated from the graph in the work of Bertelsmann and Heist. ${ }^{18}$ As expected, the value calculated from the zero velocity model is lower than that based on the stagnant background gas equations and in this case there is just over $10 \%$ difference between the two values. As noted earlier, Eq. (13) can be used to compute the velocity profile within the TDCC under the stagnant background gas assumption. In this test case, the velocity values are $\sim 0.1 \mathrm{~cm} \mathrm{~s}^{-1}$ for a chamber diameter of $10.38 \mathrm{~cm}$ and height of $1.384 \mathrm{~cm}$. In this case since 
TABLE I. Comparison between maximum supersaturation values for nonane taken from the work of Katz (column 5), calculated using the stagnant gas assumption (columns 6 and 7), and calculated with the zero velocity assumption (column 8). For each experiment, the total chamber pressure, $P_{t}$, and the temperature of the bottom, $T_{\text {bot }}$, and top, $T_{\text {top }}$, plates are shown. Experiments highlighted with an $a$ represent runs where the total pressure in the chamber was doubled.

\begin{tabular}{|c|c|c|c|c|c|c|c|}
\hline Expt. No. & $P_{t}($ torr $)$ & $T_{\text {bot }}(\mathrm{K})$ & $T_{\text {top }}(\mathrm{K})$ & $S_{\max }$ & $S_{\max }$ & $S_{\max }$ & $S_{\max }$ \\
\hline Soret/Dufour Included? & & & & Yes & Yes & No & No \\
\hline$N_{B z}=0$ used? & & & & Yes & Yes & Yes & No \\
\hline Source & & & & Katz $^{1}$ & This work & This work & This work \\
\hline 6 & 340.0 & 343.61 & 262.79 & 13.46 & 13.52 & 13.15 & 6.43 \\
\hline $6^{a}$ & 659.0 & 346.66 & 262.80 & 13.72 & 13.65 & 13.22 & 7.51 \\
\hline 11 & 95.9 & 319.65 & 243.12 & 26.54 & 26.68 & 25.93 & 11.59 \\
\hline 15 & 45.0 & 305.59 & 231.90 & 41.89 & 42.11 & 40.82 & 17.88 \\
\hline $15^{a}$ & 89.4 & 307.54 & 231.84 & 41.50 & 41.26 & 39.83 & 21.30 \\
\hline
\end{tabular}

the velocity values are small, it is not surprising that the two models give reasonably close values for the maximum supersaturation.

A second comparison is made between the values calculated by Katz for a nonane-helium system. ${ }^{9}$ In this case, a direct comparison is more complicated because Katz included the Soret/Dufour effects on the profiles. Fortunately, Katz also included a detailed sensitivity analysis for these same data and examined the effect of several parameters upon the maximum calculated supersaturation anywhere within the chamber. His analysis indicates that the effect of neglecting these coupling terms causes an approximately $3 \%$ deviation in the maximum supersaturation.

Table I is a comparison between the values derived by Katz, the values calculated via the stagnant background gas approximation (with and without Soret/Dufour terms) and the $S_{\max }$ calculated with the zero velocity approximation. As is seen in the table, the results calculated via our stagnant gas model are essentially identical when the Soret/Dufour effects are included. When these effects are not included the results are consistent with a $3 \%$ variation in the values as noted by Katz. This reinforces the fact that the physical properties and solution procedure are consistent with those used by Katz. The values for the supersaturation calculated with the zero velocity approximation are much lower; approximately $50 \%$ lower for most of the cases shown. However, there is a very real concern as to the reliability of all the low temperature critical supersaturation data for nonane.

The mass flux of the background gas, $n_{B}$, with respect to a stationary coordinate system is given by

$$
n_{B}=j_{B}+\rho w_{B} v=-\rho D_{A B} \nabla w_{B}+\rho w_{B} v
$$

and is composed of a diffusive flux term, $j_{B}$, and a convective term. The assumption of a stagnant background gas, $n_{B}=0$, means that the convective term (the so-called Stefan flow), is exactly balanced by the diffusive term. In short, even though a concentration gradient exists in the background gas, the background gas remains stagnant because there is a compensating convective flux which exactly cancels this diffusive flux. When the concentration gradient is large, (e.g., when the total pressure is decreased), this Stefan flow term is also large. Some of these velocities can become quite large. For example, in case 11 of Table I these velocities range from $\sim 3-14 \mathrm{~cm} \mathrm{~s}^{-1}$ for a chamber height of 5.27 $\mathrm{cm}$.

Katz obtained excellent agreement between the experimentally measured critical supersaturations (using the equations based on the stagnant gas approximation) for nonane and classical nucleation theory (CNT) so discrepancies between these new equations and CNT are likely based on the sample of results in Table I. The $S_{\mathrm{cr}}$ vs $T$ envelopes from Katz' experimental data are plotted in Fig. 6 as short dashes along with the predictions of CNT using the physical properties for nonane given in the original work. As shown in the figure, the agreement between the two is excellent. The experimental data are a bit below CNT predictions at the lower temperature end and a bit higher at the higher temperature end-this behavior is similar to that seen with a large number of other materials in the TDCC. It is important to point out that all of the supersaturation versus temperature curves shown in Fig. 6, except for the five curves in each set at the

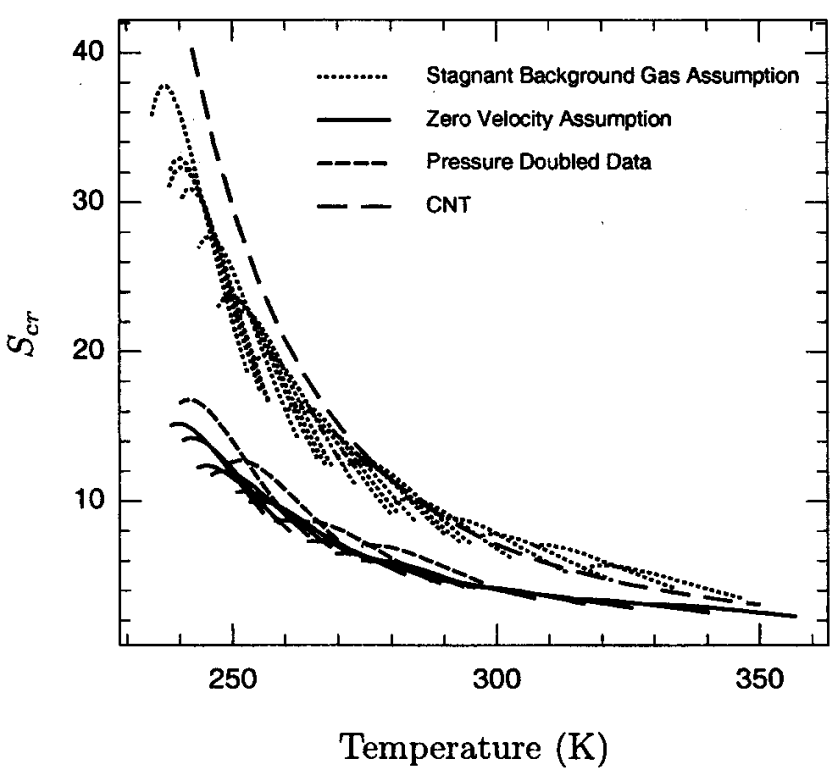

FIG. 6. A comparison between nonane experimental data and CNT predictions. The supersaturation prediction based on the zero velocity assumption indicates a pressure effect while the typical equations used to calculate the supersaturation profile in the TDCC do not show this effect. 
highest temperatures, violate the stability criteria for stable (e.g., free from buoyancy-driven convective flows) TDCC operation as defined by Bertelsmann and Heist. ${ }^{6,18}$ As a result, all these data are suspect and should most probably be represented by smaller computed supersaturation values. ${ }^{7,18}$ However, the reduction in the computed supersaturation due to the buoyancy-driven flow in this case is not as significant as the reduction in the computed supersaturation arising from the two models discussed here.

The $S_{\text {сг }}$ vs $T$ envelopes based on the general equations where the zero velocity assumption is made are shown as the solid curves. These data fall well below the CNT predictions. At the lower temperature end, the $S_{\mathrm{cr}}$ values are less than $1 / 2$ of those of the Katz and CNT predictions and the differences between the two become smaller at the higher temperatures. Another important point to notice is that the data with the stagnant background gas yield a smooth $S_{\mathrm{cr}}$ vs $T$ envelope. In contrast, there are four data sets for the newly calculated data which are markedly higher than the rest of the data set. These four sets correspond to runs where the pressure in the TDCC was doubled.

As noted in the introduction, one of the problems currently plaguing TDCC work is the apparent dependence of the results upon the pressure of the chamber carrier gas. Such an effect is not predicted by CNT or the typical equations used to calculate the supersaturation profile in the chamber. Yet the transport equations under the zero velocity assumption in this work predict a rise in the supersaturation with increasing pressure.

Again, we restrict our discussion to concentrationinduced diffusion and ignore the smaller-order, crosscoupling terms in the mass and energy equations. The equations describing mass transport under both models are of the form

$$
\frac{d}{d z}\left[\Gamma \frac{d \phi}{d z}\right]=0 .
$$

When steady conditions are reached in the TDCC, the temperature of the upper and lower plates fix the partial pressure at these boundaries via the vapor pressure equation. These partial pressures are essentially invariant with pressure, so the $\phi$ 's in Eq. (25), whether they are mass or mole fractions scale accordingly with pressure-they are both simply different ways of describing the partial pressure profile in the chamber. Variations in the partial pressure profile occur between these two fixed boundary conditions because of the factor, $\Gamma$, in Eq. (25). For Eq. (19),

$$
\Gamma=\left(\frac{c D_{A B}}{1-x_{A}}\right) .
$$

The mole fractions in TDCC experiments are typically small. For example, the largest mole fraction of any of the examples shown in Table $\mathrm{I}$ is 0.15 . Therefore, the denominator in Eq. (26) is $\sim 1.0$. Further, the product of $c D_{A B}$ is essentially pressure independent. Hence, a doubling of pressure would make little difference to the supersaturation profile calculated using Eq. (20).

On the other hand, in Eq. (15),

$$
\Gamma=\rho D_{A B} .
$$

In this case a doubling of the pressure effectively doubles this value, thereby altering the calculated supersaturation profile. Equation (23) predicts an increase in the supersaturation with pressure, although the direction of this change (an increasing maximum supersaturation with increasing pressure) is opposite to that which is typically observed.

As a brief aside, we note that Eq. (26) will become large as the mole fraction of the diffusing component approaches unity. The region in the TDCC where this will occur is in the vicinity of the lower plate boundary, and the reason why this would occur is operating the cloud chamber under low total pressure conditions. As mentioned earlier, typical values for mole fractions at the lower plate are usually small (several tenths or less), so this is normally not an issue. However, operation under conditions in which the value of the vapor pressure of the diffusing component at the lower plate approaches the magnitude of the total pressure is becoming increasingly important as the cloud chamber is being used to investigate broader classes of working fluids over wider ranges of operational conditions (e.g., nucleation near a critical point ${ }^{19}$ ).

In such experiments, as the value of $x_{A}$ approaches unity the value of the mass flux will increase significantly and the conditions at the lower plate surface will move increasingly away from equilibrium. When that happens, we are no longer able to use the equilibrium boundary condition approximation for the mole fractions at the lower (and upper) plate surfaces, and we are no longer able to calculate conditions within the chamber. Again, this is generally not a problem as long as the ratio of the mass flux through the chamber to the equilibrium evaporation flux at the lower plate is small. For example, in recent experiments involving pentanol and hydrogen in which operation at low total pressures was specifically investigated, this ratio was typically on the order of $10^{-6}$ (even at the lower total pressures used in those experiments ${ }^{20}$ ).

In the past, investigators have relied on empirical rules of thumb to help determine proper operating ranges for the TDCC. One such rule involves the so-called pressure ratio. This quantity is defined as the ratio of the total pressure to the equilibrium vapor pressure of the diffusing material at the lower plate. It is generally accepted that the value of this ratio should be larger than (roughly) two to three and the bigger the better. ${ }^{21}$ At first glance it might seem reasonable to associate the effect of the denominator in Eq. (26) with this pressure ratio.

However, results from the pentanol-hydrogen investigation mentioned above clearly identified a lower total pressure stability limit for diffusion cloud chamber operation below which the nucleation data are increasingly unreliable. ${ }^{20}$ In that investigation, mole fraction and temperature profiles within the cloud chamber were determined using the stagnant background gas assumption (including thermal diffusion cross coupling terms and using a real gas equation of state). In that investigation, the ratio of the mass flux to the equilibrium flux was generally of the order $10^{-6}$. Based on the results of that investigation, it does not appear that the 
observed limit of lower total pressure stability reported in the pentanol-hydrogen investigation is a consequence of the denominator in Eq. (26). Rather, as the authors point out, it appears to be associated with the onset of buoyancy-driven convective instabilities within the cloud chamber and appears also to be related to the presence of the thin pool of liquid (source of diffusing vapor) on the lower plate. One other important result of that investigation is that the pressure ratio bears no relation to the lower total pressure limit of stability and should not be used to specify operational conditions for diffusion cloud chamber operation.

\section{CONCLUSIONS}

As noted in the introduction, recent diffusion cloud chamber experiments seem to indicate that measured critical supersaturations depend upon both the type and pressure of the background gas, even though such an effect does not appear in the equations typically used in the TDCC data reduction. In this work we have tried to highlight differences between two different 1D models of the TDCC - the typical stagnant gas approximation and one based on purely diffusional transport. This second model is based on the assumption of no mass-average velocities within the chamber and results in an incorrect boundary condition for the flux of the background gas. Several inconsistencies have been noted for the Stefan tube problem and these same arguments are also applicable to the stagnant background gas model of the TDCC. As expected, for situations where the Stefan flow is very small, the two models give similar results. As these velocities become much higher, critical supersaturations for these two models can differ by as much as $50 \%$. One interesting feature of the zero velocity model is that it predicts a rise in the critical supersaturation with pressure, although it should be noted that the direction of this change is opposite to that which is observed experimentally. The limitations of each of these $1 \mathrm{D}$ models may be resolved with a $2 \mathrm{D}$ model of the chamber and it would be interesting to compare such a solution with these 1D predictions.

${ }^{1}$ J. L. Katz and B. J. Ostermier, J. Chem. Phys. 47, 478 (1967).

${ }^{2}$ Y. Viisanen, R. Strey, and H. Reiss, J. Chem. Phys. 99, 4680 (1993).

${ }^{3}$ R. H. Heist, M. Janjua, and J. Ahmed, J. Phys. Chem. 98, 4443 (1994)

${ }^{4}$ R. H. Heist, J. Ahmed, and M. Janjua, J. Phys. Chem. 99, 375 (1995).

${ }^{5}$ R. H. Heist, J. Phys. Chem. 99, 16792 (1995).

${ }^{6}$ A. Bertelsmann and R. H. Heist, J. Chem. Phys. 106, 624 (1997).

${ }^{7}$ F. T. Ferguson and J. A. Nuth III, J. Phys. Chem. 111, 8013 (1999).

${ }^{8}$ R. B. Bird, W. E. Stewart, and E. N. Lightfoot, Transport Phenomena (Wiley, New York, 1960).

${ }^{9}$ J. L. Katz, J. Chem. Phys. 52, 4733 (1970).

${ }^{10} \mathrm{~S}$. Whitaker, Ind. Eng. Chem. Res. 30, 978 (1991)

${ }^{11}$ F. J. Heinzelmann, D. T. Wasan, and C. R. Wilke, Ind. Eng. Chem. Fundam. 4, 55 (1965).

${ }^{12}$ S. S. Rao and C. O. Bennett, Ind. Eng. Chem. Res. 5, 573 (1966).

${ }^{13}$ H. A. Kramers and J. Kistemaker, Physica (Amsterdam) 10, 699 (1943).

${ }^{14}$ S. Whitaker, Ind. Eng. Chem. Fundam. 6, 476 (1967).

${ }^{15}$ S. S. Rao and C. O. Bennett, Ind. Eng. Chem. Fundam. 6, 477 (1967).

${ }^{16}$ J. P. Meyer and M. D. Kostin, Int. J. Heat Mass Transf. 18, 1293 (1975).

${ }^{17}$ B. L. Markham and F. Rosenberger, Chem. Eng. Commun. 5, 287 (1980).

${ }^{18}$ A. Bertelsmann and R. H. Heist, J. Chem. Phys. 106, 610 (1997).

${ }^{19}$ A. Bertelsmann, P. Ye, and R. H. Heist (unpublished).

${ }^{20}$ R. H. Heist, A. Bertelsmann, D. Martinez, and Y. F. Chan (unpublished).

${ }^{21}$ J. L. Katz, C. J. Scoopa II, N. G. Kumar, and P. Mirabel, J. Chem. Phys. 62, 448 (1975). 